\title{
Simultaneous Improvement of Formaldehyde Emission and Adhesion of Medium-Density Fiberboard Bonded with Low-Molar Ratio Urea-Formaldehyde Resins Modified with Nanoclay ${ }^{1}$
}

\author{
Eko Setio $\mathrm{WIBOWO}^{2} \cdot$ Muhammad Adly Rahandi LUBIS $^{3} \cdot$ Byung-Dae PARK $^{2, \dagger}$
}

\begin{abstract}
In wood-based composite panels, low-molar ratio (LMR) urea-formaldehyde (UF) resins usually result in reduced formaldehyde emission (FE) at the expense of poor adhesion. However, the FE and adhesion of medium-density fiberboard (MDF) bonded with LMR UF resins were both improved in this study. The modified LMR UF resins with transition metal ion-modified bentonite (TMI-BNT) nanoclay simultaneously improved the FE and adhesion of MDF panels. The modified LMR UF resins with $5 \%$ TMI-BNT resulted in a $37.1 \%$ FE reduction and $102.6 \%$ increase in the internal bonding (IB) strength of MDF panels. Furthermore, thickness swelling and water absorption also significantly decreased to $13.0 \%$ and $24.9 \%$, respectively. These results imply that TMI-BNT modification of LMR UF resins could enhance the formation of a three-dimensional network rather than crystalline domains, resulting in improved cohesion.
\end{abstract}

Keywords: formaldehyde emission, low-molar ratio, urea-formaldehyde resins, medium-density fiberboard, nanoclay

\section{INTRODUCTION}

Medium-density fiberboard (MDF) is a wood-based composite panel fabricated by combining wood fibers with a binder and forming them into panels by hotpressing under high temperature and pressure (Thoemen et al., 2010). It was first produced commercially in 1965 in the United States and is still considered a premium product in the wood-based panel industry (Lubis et al., 2018). In the last few decades, the production of MDF panels has increased rapidly due to their ad- vantages over solid wood, including uniform and smooth surfaces, high dimensional stability, ease of modification for various purposes, and provision of an excellent substrate for decorative overlays or furniture products (Çöpür et al., 2008). MDF properties depend on several factors, such as fiber quality, processing parameters, hot-pressing conditions, and resin adhesives. Of these factors, adhesive parameters are considered as one of the most important aspects because resin adhesive represents at least $29 \%$ of the total cost in MDF manufacture (Hong et al., 2017). Resin should have

${ }^{1}$ Date Received March 27, 2021, Date Accepted July 21, 2021

2 Department of Wood and Paper Science, Kyungpook National University, Daegu, 41566, Republic of Korea

${ }^{3}$ Research Center for Biomaterials, Indonesian Institute of Sciences (LIPI), Jl. Raya Bogor Km. 46, Cibinong, Bogor 16911, Indonesia

† Corresponding author: Byung-Dae PARK (e-mail: byungdae@knu.ac.kr, ORCID: 0000-0002-9802-7855) 
a sufficient cure at the end of hot-pressing (Hong et al., 2017). This and the reactivity, mobility, and bond strength of resins are crucial for producing MDF with excellent physical and mechanical performances.

One of the most widely used resin for MDF production is the urea-formaldehyde (UF) resin. For several years, many researchers conducted various research on the use of amino resins for wood-based panels (Han et al., 2019; Jeong et al., 2020; Lubis et al., 2019b, 2019a; Wen et al., 2020). UF resins among the amino resins are probably the most suitable for MDF due to their excellent properties, including fast curing time, good bonding strength, clear color, and low production cost (Dunky, 1998; Jeong and Park, 2019; Lubis and Park, 2020a; Park and Park, 2021). Despite their advantages, UF resins emit formaldehyde emission (FE) when used for an MDF panel, which is a critical disadvantage, and harmful to environmental and human health. Hence, lowering the formaldehyde-to-urea $(\mathrm{F} / \mathrm{U})$ molar ratio was employed to significantly reduce the FE (Meyer et al., 1985; Myers, 1984; Pizzi and Valenzuela, 1994). However, by decreasing the $\mathrm{F} / \mathrm{U}$ molar ratio to 1.0 or less, a significant amount of crystalline domains is formed instead of a tridimensional cross-linked network structure, which consequently decreases the cohesion and bonding strength (Levendis et al., 1992; Park and Jeong, 2011; Park et al., 2006). For some years, hydrogen bonding between linear molecules was reported as responsible for the formation of crystalline domains in low-molar ratio (LMR) UF resins (Dunker et al., 1986; Stuligross and Koutsky, 1985; Wibowo et al., 2020b). Linear molecules are formed because of the cleavage of the oxymethylene and branched methylene ether linkages by the excess urea added after the condensation reaction (Wibowo et al., 2020b; Wibowo and Park, 2021). Hence, the crystalline domains should be reduced by preventing hydrogen between linear molecules to obtain the low FE and high bonding strength of LMR UF resins.

Our recent studies revealed that the incorporation of 5\% TMI-BNT nanoclay or $20 \%$ melamine during the synthesis of $1.0 \mathrm{~F} / \mathrm{U}$ molar-ratio UF resins successfully has converted semi-crystalline UF resins to amorphous polymers (Wibowo et al., 2020a; Wibowo and Park, 2020), and led to better adhesive performance when they were used as adhesives for plywood. However, the application of these modified UF resins to other wood-based materials, such as MDF, has not yet been clarified. Thus, this study attempts to simultaneously improve the FE and adhesion of MDF panels bonded with LMR UF resins modified with TMI-BNT nanoclay at different levels.

\section{MATERIALS and METHODS}

\subsection{Materials}

The bentonite clay (montmorillonite) used in this work was purchased from Sigma Aldrich. $\mathrm{NaCl}$, anhydrous $\mathrm{CuCl}_{2}$, formalin (37\%), and extra pure-grade urea (99 wt.\%) were obtained from Daejung Chemical, Republic of Korea. The extra pure-grade $\mathrm{NH}_{4} \mathrm{Cl}$ (99 wt.\%), $\mathrm{NaOH}$ (93 wt.\%), and aqueous formic acid (85\%) were purchased from Duksan Chemical, Republic of Korea. High-performance liquid chromatography (HPLC)grade dimethylformamide (DMF, 99.9\%) and dimethyl sulfoxide (DMSO, 99.9\%) used as the mobile phase for gel permeation chromatography analysis were obtained from Fisher Scientific and Duksan Chemical Korea, respectively.

\subsection{Synthesis and properties of modified LMR UF resins with different TMI-BNT levels}

The TMI-BNT nanoclay was prepared using the ion exchange method, as reported in a previous work (Wibowo et al., 2020a). The obtained TMI-BNT was used for the in situ modification of the $1.0 \mathrm{~F} / \mathrm{U}$ molar-ratio UF resins synthesized via an alkaline-acid 
Simultaneous Improvement of Formaldehyde Emission and Adhesion of Medium-Density Fiberboard Bonded with Low-Molar Ratio Urea-Formaldehyde Resins Modified with Nanoclay

reaction. Different TMI-BNT levels of $0 \%, 1 \%, 3 \%$, and $5 \%$ based on the theoretical solid content of UF resins were added to formalin in a glass vessel. The temperature and $\mathrm{pH}$ were then adjusted to $90^{\circ} \mathrm{C}$ and 8.0 , respectively. Subsequently, the formalin solution was poured into a glass reactor, adjusted to $\mathrm{pH} 8.0$ using sodium hydroxide $(\mathrm{NaOH}, 20$ wt.\%), and then heated to $40^{\circ} \mathrm{C}$. Urea was subsequently added to the reactor to give an initial $\mathrm{F} / \mathrm{U}$ molar ratio of 2.0. The mixture was then heated to $90^{\circ} \mathrm{C}$, followed by the addition of different levels of the prepared TMI-BNT solution. Furthermore, the $\mathrm{pH}$ was maintained at 8.0 for $1 \mathrm{~h}$ to allow the addition reaction to proceed. And then, the temperature and $\mathrm{pH}$ were adjusted to $80^{\circ} \mathrm{C}$ and 4.6, respectively, to initiate the condensation reaction. The reaction was terminated when a target viscosity of the " $\mathrm{J}-\mathrm{K}$ " scale was reached based on the readings using a bubble viscometer (VG 900, GardenerHoldt, Columbia, South Carolina, USA). A certain amount of urea was added to the glass reactor to obtain a final $\mathrm{F} / \mathrm{U}$ molar ratio of 1.0. The temperature was maintained at $60^{\circ} \mathrm{C}$ for $20 \mathrm{~min}$. Subsequently, the resins were cooled to $25^{\circ} \mathrm{C}$ and the $\mathrm{pH}$ was adjusted to $\sim 8.2$. The resins were then stored at room temperature for $24 \mathrm{~h}$ prior to their analysis. The viscosity, gelation time, and apparent molecular weights $\left(M_{\mathrm{w}}\right)$ of the modified UF resins with different TMI-BNT levels were determined as per the method used in a previous work. (Wibowo et al., 2020a)

\subsection{Preparation and determination of MDF properties}

Six single-layer MDF panels were prepared using modified UF resins with different TMI-BNT levels (i.e., $1 \%, 3 \%$, and $5 \%$; two panels for each level). Approximately $12 \%$ modified resins and $1 \%$ emulsion wax based on the dry mass of wood fibers were prepared in a $1000 \mathrm{~mL}$ beaker glass. Furthermore, 3\%
$\mathrm{NH}_{4} \mathrm{Cl}$ was added to the beaker glass based on the resin solid content. The mixture was then sprayed onto the fibers in a drum-type mechanical blender. The modified resins and emulsion wax were fed into a 1.4 mm-diameter atomization nozzle using a peristaltic pump at $50 \mathrm{rpm}$ and $196 \mathrm{kPa}$ air pressure in the blender. The resinated fibers were used to prepare the fiber mat using a centrifugal air blower. This fiber mat was prepressed for $30 \mathrm{~s}$ and hot-pressed at $180^{\circ} \mathrm{C}$ using a single-phase pressing schedule of $7.85 \mathrm{MPa}$ for $240 \mathrm{~s}(20 \mathrm{~s} / \mathrm{mm})$ to produce MDF panels $(300 \mathrm{~mm}$ $\times 400 \mathrm{~mm} \times 12 \mathrm{~mm}$ ). The final thickness was adjusted to $12 \mathrm{~mm}$ using two stop-bars in the hot-press. As a control, two MDF panels with $700 \mathrm{~kg} / \mathrm{m}^{3}$ density and $12 \mathrm{~mm}$ thickness were made using neat UF resins. All MDF panels were evaluated according to published methods (Hong et al., 2020; KS F3200, 2016; Lubis et al., 2020). For mechanical properties, eight samples $(50 \mathrm{~mm} \times 50 \mathrm{~mm} \times 12 \mathrm{~mm})$ were prepared to determine the internal bonding (IB) strength, and three samples $(200 \mathrm{~mm} \times 50 \mathrm{~mm} \times 12 \mathrm{~mm})$ were used to obtain the modulus of rupture (MOR) and modulus of elasticity (MOE). The IB strength, MOR, and MOE of the MDF panels were evaluated using the universal testing machine at cross-head speeds of $2 \mathrm{~mm} / \mathrm{min}, 10$ $\mathrm{mm} / \mathrm{min}$, and $10 \mathrm{~mm} / \mathrm{min}$, respectively. Furthermore, ten samples $(50 \mathrm{~mm} \times 50 \mathrm{~mm} \times 12 \mathrm{~mm})$ were soaked for $24 \mathrm{~h}$ in water, and then their thicknesses and weights were measured after removing the surface water for the thickness swelling (TS) and water absorption (WA) tests. According to the desiccator method, ten MDF samples $(150 \mathrm{~mm} \times 50 \mathrm{~mm} \times 12 \mathrm{~mm})$ were placed in a desiccator for $24 \mathrm{~h}$, and then UV-visible spectrophotometry (Optizen 3220 UV, Mecasys, Daejeon, Korea) was used at a wavelength of $412 \mathrm{~nm}$ to determine the formaldehyde emission (FE) values. A statistical analysis was performed to evaluate the MDF properties bonded with the modified UF resins with different TMI-BNT levels using the analysis of 
variance and the Duncan multiple range test at $\alpha=0.05$. The SPPS 25 (SPSS Inc., Chicago, USA) software package was used for the calculations.

\section{RESULTS and DISCUSSION}

\subsection{Properties of modified UF resins with different TMI-BNT levels}

Table 1 presents the properties of the modified UF resins with different TMI-BNT levels. The addition of the TMI-BNT improved the resin properties of the LMR UF resins. For example, the gelation time decreased as the TMI-BNT content increased, indicating that the TMI-BNT facilitated the cure reaction of UF resins. As shown in Scheme 1, TMI-BNT can provide acids that possibly arise from the complexation of $\mathrm{Cu}^{2+}$ and surface hydroxyl groups on the nanoclay (Vico, 2003). The released protons $\left(\mathrm{H}^{+}\right)$could accelerate the condensation reaction, resulting in a lower gelation time. (Sun et al., 2014) In agreement with the gelation time results, viscosities and molecular weights of the samples increased as the TMI-BNT content increased from $1 \%$ to $5 \%$. This indicates that the modified UF resins with TMI-BNT were composed of bigger molecules compared to the neat UF resin, which is attributed to the formation of more branched molecules in the former resin. As described in (Wibowo et al., 2020a), the branched polymer formation was

Table 1. Properties of the modified UF resins with different TMI-BNT levels

\begin{tabular}{cccc}
\hline $\begin{array}{c}\text { TMI-BNT content } \\
(\%)\end{array}$ & $\begin{array}{c}\text { Gelation time } \\
(\mathrm{s})\end{array}$ & $\begin{array}{c}\text { Viscosity } \\
(\mathrm{mPa} . \mathrm{s})\end{array}$ & $\begin{array}{c}M_{\mathrm{w}} \\
(\mathrm{g} / \mathrm{mol})\end{array}$ \\
\hline \hline $0 *$ & 204 & 240.2 & 425 \\
1 & 194 & 250.2 & 485 \\
3 & 192 & 252.2 & 526 \\
5 & 154 & 300.0 & 580 \\
\hline
\end{tabular}

* The data were taken from the previous work (Wibowo et al., 2020a). induced by the TMI-BNT intercalation between linear molecules (Scheme 1), resulting in the crystallinity reduction caused by the hydrogen bonding prevention. As the result of blocked hydrogen bonding, the linear molecules could easily conduct a condensation reaction and lead to the formation of a more branched structure compared to the neat UF resins. In addition, the viscosity and molecular weight increased as the TMI-BNT content increased from $1 \%$ to $5 \%$ (Table 1). Furthermore, the UF resin crystallinity decreased from $52.3 \%$ to $35.2 \%$ as the TMI-BNT content increased from $0 \%$ to $5 \%$ and transformed the semicrystalline XRD pattern of the low-molar ratio UF resins to amorphous, similar to the high-molar-ratio resins (Wibowo et al., 2020a). Moreover, (Wibowo et al., 2020a) demonstrated that the cross-linking density $\left(V_{\mathrm{e}}\right)$ increased as the TMI-BNT level increased from $0 \%$ to $5 \%$, regardless of isothermal temperatures, and confirmed that the TMI-BNT facilitated the formation of branched polymer through interaction (Scheme 1). As a result, the branched polymer of modified UF resins showed more degrees of cross-linking after curing, resulting in an amorphous structure. Similarly, the decline in the crystallinity from $52.3 \%$ to $22.0 \%$ oc-

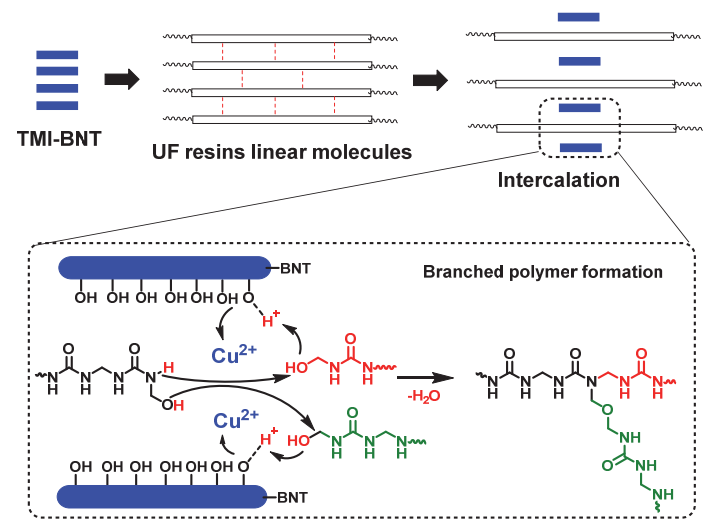

Scheme 1. Schematic diagram of the intercalation of TMI-BNT in LMR UF resins, proton release from the TMI-BNT, and typical acid-catalyzed condensation of UF resins. 
Simultaneous Improvement of Formaldehyde Emission and Adhesion of Medium-Density Fiberboard Bonded with Low-Molar Ratio Urea-Formaldehyde Resins Modified with Nanoclay

curred when $20 \%$ of melamine was introduced to LMR UF resins, resulting in the significant increase of $V_{\mathrm{e}}$ (Wibowo and Park, 2020). In this case, melamine having three active $-\mathrm{NH}_{2}$ groups can react with formaldehyde to form six hydroxymethyl groups and lead to the formation of a highly branched structure. Consequently, hydrogen bonding between linear molecules in LMR resins can be prevented as the linear molecules separated from each other, resulting in a significant decrease in crystallinity. This result indicates a strong relationship between the crystallinity of UF resins and their degree of cross-linking. It appears that a decrease in the crystallinity of semicrystalline polymers can be considered as one indication of the formation of branched chains in polymer structure as the linear structures that form ordered arrangement through hydrogen bonding, particularly for the case of LMR UF resins.

\subsection{Performance of the MDF panels bonded with modified UF resins}

Fig. 1 depicts the properties of the MDF panels bonded with the modified UF resins at different TMI-BNT levels. The properties of the MDF bonded with the modified UF resins are generally better than those of neat UF resins, especially at the $5 \%$ TMI-BNT level. For example, the IB strength significantly increased from $\sim 0.39$ to $\sim 0.79 \mathrm{MPa}$ when the TMI-BNT level was increased to 5\% (Fig. 1(a)), which is consistent with the increase in the cross-linking density and molecular weight and the decrease in the gelation time. This result agrees with previous results, which used 5\% octadecylamine-modified bentonite (ODA-BNT) and TMI-BNT for the LMR UF resins to enhance plywood performance (Lubis and Park, 2020b; Wibowo et al., 2020a). Another research mentioned that adding $8 \%-10 \% \mathrm{Na}$ montmorillonite (NaMMT) in UF resins with $1.1 \mathrm{~F} / \mathrm{U}$ molar ratio could increase the IB strength of the particleboard bonded with them (Lei et al., 2008). The results also proved that the intercalation of the linear molecules of the UF resins between the clay layers reduced the crystalline domains by preventing hydrogen bonding between the linear molecules, which resulted in a stronger cohesion caused by the easier formation of a cross-linked structure when the resins were cured. Moreover, the FE reduced from $\sim 0.60$ to $\sim 0.38 \mathrm{mg} / \mathrm{L}$ when $5 \%$ TMIBNT was added (Fig. 1(b)). The intercalated and exfoliated nanoclay had an extremely high aspect ratio and a specific surface area, which enabled them to react easily with the active functional groups of neat resins, leading to an improvement in bonding strength and a decrease of the FE (Salari et al., 2012). In addition, the abundant hydroxyl groups on the nanomaterial surface can react with the free formaldehyde and subsequently decrease the FE (Cademartori et al., 2019; Salari et al., 2012). However, the results of the MOR and MOE statistically showed that all modified UF resins had no significantly different properties compared to that of neat UF resins (Fig. 1(c)-(d)). In other words, the MOR and MOE of the MDF panels were more likely to be affected by the wood fiber characteristics, including the fiber size distribution, bulk density, $\mathrm{pH}$ value, and buffering capacity (Xing et al., 2006), than by the binder itself. Furthermore, the TMI-BNT appeared to have significantly improved the water resistance of the MDF panels, especially with $5 \%$ content, which was possibly because of the improved IB strength. This was also proven by the fact that the TS and WA were decreased to $\sim 13 \%$ and $\sim 25 \%$, respectively, as the TMI-BNT content increased to $5 \%$ (Fig. 1(e)-(f)). The hypothesis regarding the improvement of the wet performance of the UF resins by montmorillonite addition has been explained in detail in the literature (Lei et al., 2008). The reference mentioned two main aspects, that is, the water-repellent nature of montmorillonite and the percolation 

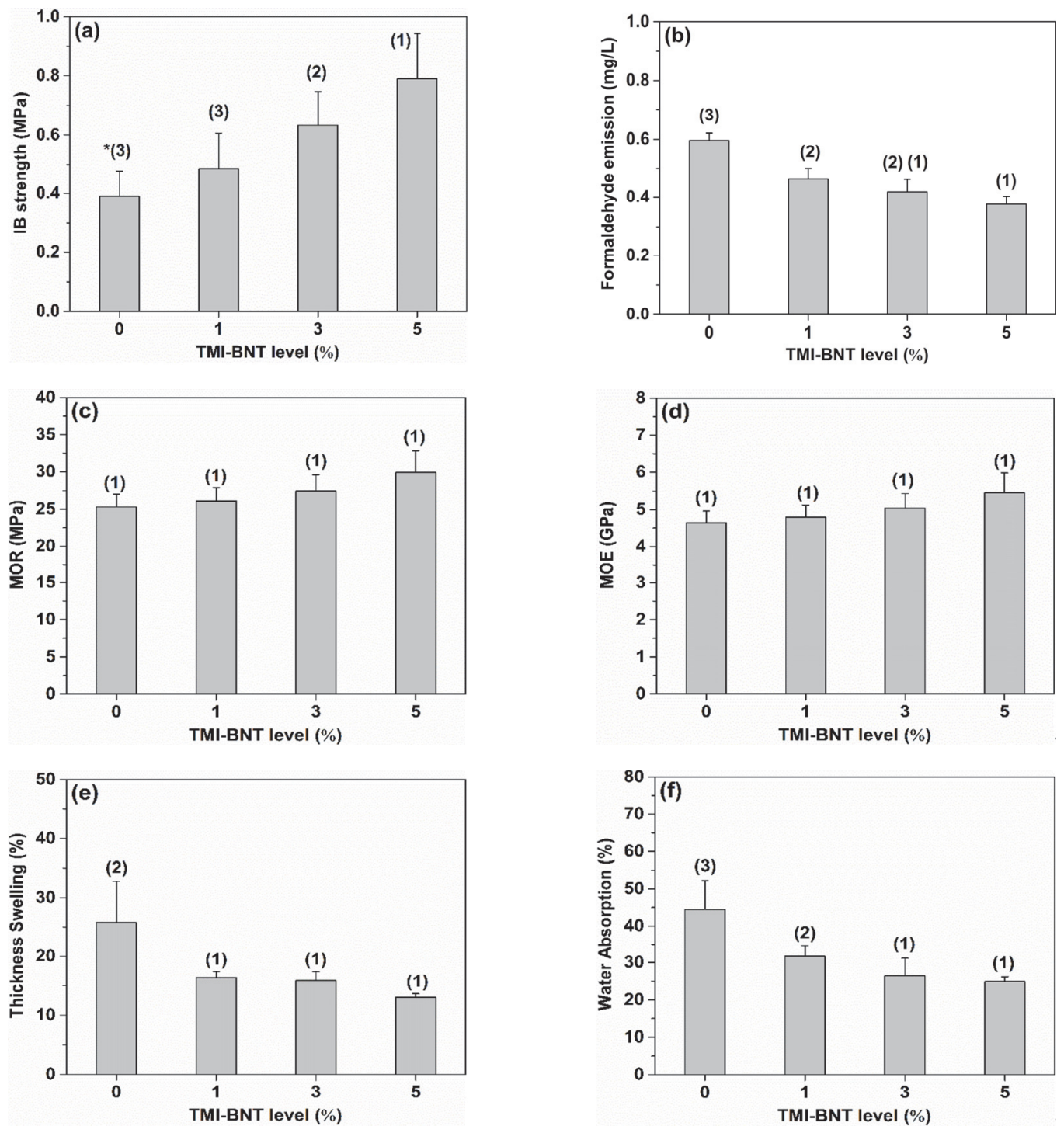

Fig. 1. Properties of the MDF bonded with the modified UF resins at different TMI-BNT levels: (a) IB strength; (b) FE; (c) MOR; (d) MOE; (e) TS; and (f) WA. *The same numbers in parenthesis are not significantly different at a $p$ value of 0.05 .

phenomenon between the nanoclay particles, which causes the observed reinforcement effect.

\section{CONCLUSION}

This study showed a simultaneous improvement of the FE and adhesion of the MDF bonded with the LMR UF resins modified with the TMI-BNT nanoclay. The modification of the LMR UF resins generally resulted in high viscosity and cross-linking density, shorter gelation time, and greater molecular weight. The FE and adhesion of the MDF panels 
Simultaneous Improvement of Formaldehyde Emission and Adhesion of Medium-Density Fiberboard Bonded with Low-Molar Ratio Urea-Formaldehyde Resins Modified with Nanoclay

bonded with the modified UF resins were significantly improved by $37.1 \%$ and $102.6 \%$, respectively, when the TMI-BNT level was increased to $5 \%$. Both the TS and WA significantly decreased to $13.0 \%$ and $24.9 \%$, respectively. These results suggest that the TMI-BNT modification enhances the formation of a three-dimensional network structure, instead of ordered crystalline domains in LMR UF resins, which leads to an increase in cohesion.

\section{ACKNOWLEDGMENT}

This work was supported by the National Research Foundation of Korea funded by the Korean Government (MSIT) (Grant No. 2020R1A2C1005042).

\section{REFERENCES}

Cademartori, P.H.G., de, Artner, M.A., Alves de Freitas, R., Magalhães, W.L.E. 2019. Alumina nanoparticles as formaldehyde scavenger for urea-formaldehyde resin: Rheological and in-situ cure performance. Compososite Part B Engineering 176.

https://doi.org/10.1016/j.compositesb.2019.107281

Çöpür, Y., Güler, C., Taşçioğlu, C., Tozluoğlu, A. 2008. Incorporation of hazelnut shell and husk in MDF production. Bioresource Technology 99(15): 7402-7406.

Dunker, A.K., John, W.E., Rammon, R., Farmer, B., Johns, S.J. 1986. Slightly bizarre protein chemistry: Urea-formaldehyde resin from a biochemical perspective. Journal of Adhesion 19(2): 153-176.

Dunky, M. 1998. Urea-formaldehyde (UF) adhesive resins for wood. International Journal of Adhesion and Adhesives 18(2): 95-107.

Han, H.J., Lee, S.E., Yang, S.M., Choi, C., Kang, S.G. 2019. Evaluation of formaldehyde emission from wood-based panels using accelerated collection method. Journal of the Korean Wood Science and
Technology 47(2): 129-144.

Hong, M.-K., Lubis, M.A.R., Park, B.-D., Sohn, C.H., Roh, J. 2020. Effects of surface laminate type and recycled fiber content on properties of three-layer medium density fiberboard. Wood Material Science \& Engineering 15(3): 163-171.

Hong, M.K., Lubis, M.A.R., Park, B.D. 2017. Effect of panel density and resin content on properties of medium density fiberboard. Journal of the Korean Wood Science and Technology 45(4): 444-455.

Jeong, B., Park, B.D. 2019. Performance of ureaformaldehyde resins synthesized at two different low molar ratios with different numbers of urea addition. Journal of the Korean Wood Science and Technology 47(2): 221-228.

Jeong, B., Park, B.D., Causin, V. 2020. Effects of storage time on molecular weights and properties of melamine-urea-formaldehyde resins. Journal of the Korean Wood Science and Technology 48(3): 291-302.

KS F3200, 2016. "Fiberboards," Korean Standards Association, Seoul, Republic of Korea.

Lei, H., Du, G., Pizzi, A., Celzard, A. 2008. Influence of nanoclay on urea-formaldehyde resins for wood adhesives and its model. Journal of Applied Polymer Science 109(4): 2442-2451.

Levendis, D., Pizzi, A., Ferg, E. 1992. The Correlation of Strength and Formaldehyde Emission with the Crystalline/Amorphous Structure of UF Resins. Holzforschung 46(3): 263-269.

Lubis, M.A.R., Hong, M.K., Park, B.D., Lee, S.M. 2018. Effects of recycled fiber content on the properties of medium density fiberboard. European Journal of Wood and Wood Products 76(5): 1515-1526. Lubis, M.A.R., Jeong, B., Park, B.D., Lee, S.M., Kang, E.C. 2019a. Effect of synthesis method and melamine content of melamine-urea-formaldehyde resins on bond-line features in plywood. Journal of the Korean Wood Science and Technology 47(5): 
579-586.

Lubis, M.A.R., Park, B.D. 2020a. Influence of initial molar ratios on the performance of low molar ratio urea-formaldehyde resin adhesives. Journal of the Korean Wood Science and Technology 48(2): 136-153.

Lubis, M.A.R., Park, B.D. 2020b. Enhancing the performance of low molar ratio urea-formaldehyde resin adhesives via in-situ modification with intercalated nanoclay. Journal of Adhesion. https://doi.org/10.1080/00218464.2020.1753515

Lubis, M.A.R., Park, B.D., Hong, M.K. 2020. Tuning of adhesion and disintegration of oxidized starch adhesives for the recycling of medium density fiberboard. BioResources 15(3): 5156-5178.

Lubis, M.A.R., Park, B.D., Lee, S.M. 2019 b. Performance of hybrid adhesives of blocked-pMDI/ melamine-urea-formaldehyde resins for the surface lamination on plywood. Journal of the Korean Wood Science and Technology 47(2): 200-209.

Meyer, B., Hermanns, K., Smith, D.C. 1985. Formaldehyde release from urea-formaldehyde bonded wood products. Journal of Adhesion 17(4): 297-308.

Myers, G. 1984. How mole ratio of UF resin affects formaldehyde emission and other properties: A literature critique. Forest Products Journal 34(5): 34-41.

Park, B.-D., Jeong, H.-W. 2011. Hydrolytic stability and crystallinity of cured urea-formaldehyde resin adhesives with different formaldehyde/urea mole ratios. International Journal of Adhesion and Adhesives 31(6): 524-529.

Park, B.D., Kang, E.C., Park, J.Y. 2006. Effects of formaldehyde to urea mole ratio on thermal curing behavior of urea-formaldehyde resin and properties of particleboard. Journal of Applied Polymer Science 101(3): 1787-1792.

Park, S., Park, B.-D. 2021. Crystallinity of low molar ratio urea-formaldehyde resins modified with cellulose nanomaterials. Journal of the Korean Wood Science and Technology 49(2):169-180.

Pizzi, A., Valenzuela, J. 1994. Theory and practice of the preparation of low formaldehyde emission uf adhesives. Holzforschung 48(3): 254-261. https://doi.org/10.1515/hfsg.1994.48.3.254

Salari, A., Tabarsa, T., Khazaeian, A., Saraeian, A. 2012. Effect of nanoclay on some applied properties of oriented strand board (OSB) made from underutilized low quality paulownia (Paulownia fortunei) wood. Journal of Wood Science. 58(6): 513-524.

Stuligross, J., Koutsky, J.A. 1985. A Morphological study of urea-formaldehyde resins. Journal of Adhesion 18(4): 281-299.

Sun, Q.N., Hse, C.Y., Shupe, T.F. 2014. Effect of different catalysts on urea-formaldehyde resin synthesis. Journal of Applied Polymer Science 131(16): 1-7.

Thoemen, H., Irle, M., Sernek, M. 2010. Wood-Based panels: An introduction for specialist. Brunel University Press, London. England, pp. 1-94.

Vico, L.I. 2003. Acid-base behaviour and $\mathrm{Cu}^{2+}$ and $\mathrm{Zn}^{2+}$ complexation properties of the sepiolite/water interface. Chemical Geology 198(3-4): 213-222.

Wen, M.Y., Zhu, J.Z., Zhu, M., Sun, Y.X., Park, H.J., Shi, J. 2020. Research on flame retardant formaldehyde-free plywood glued by aqueous polymer isocyanate adhesive1. Journal of the Korean Wood Science and Technology 48(5): 755-764.

Wibowo, E.S., Lubis, M.A.R., Park, B.-D., Kim, J.S., Causin, V. 2020a. Converting crystalline thermosetting urea-formaldehyde resins to amorphous polymer using modified nanoclay. Journal of Industrial Engineering Chemistry 87: 78-89.

Wibowo, E.S., Park, B.-D., Causin, V. 2020 b. Hydrogen-Bond-Induced crystallization in lowmolar-ratio urea-formaldehyde resins during synthesis. Industrial \& Engineering Chemistry 
Simultaneous Improvement of Formaldehyde Emission and Adhesion of Medium-Density Fiberboard Bonded with Low-Molar Ratio Urea-Formaldehyde Resins Modified with Nanoclay

Research 59(29): 13095-13104.

Wibowo, E.S., Park, B. 2021. Crystalline lamellar structure of thermosetting urea-formaldehyde resins at a low molar ratio. Macromolecules 54(5): 2366-2375. Wibowo, E.S., Park, B. 2020. Enhancing adhesion of thermosetting urea-formaldehyde resins by preventing the formation of $\mathrm{H}$-bonds with multi-reactive melamine. Journal of Adhesion.

https://doi.org/10.1080/00218464.2020.1830069

Xing, C., Zhang, S.Y., Deng, J., Riedl, B., Cloutier, A. 2006. Medium-density fiberboard performance as affected by wood fiber acidity, bulk density, and size distribution. Wood Science and Technology 40(8): 637-646. 